\title{
Reasons for cannabis use in psychosis
}

\author{
David Schofield, Chris Tennant, Louise Nash, Louisa Degenhardt, Alison Cornish, \\ Coletta Hobbs, Gail Brennan
}

\begin{abstract}
Objective: To examine the reasons for cannabis use among individuals with psychotic disorders.

Method: Forty-nine people with psychotic disorders in treatment with community health centres in Northern Sydney were interviewed to collect information about their experience of antipsychotic side-effects and their influence on cannabis use. Other information collected on cannabis use included: amount and frequency, effects of use and other general reasons given for use.

Results: It was found that boredom, social motives, improving sleep, anxiety and agitation and symptoms associated with negative psychotic symptoms or depression were the most important motivators of cannabis use. Positive symptoms of psychosis and antipsychotic side-effects that were not associated with anxiety, were not important motivators of cannabis use.

Conclusions: As cannabis use may precipitate relapse in this population, it is important to reduce these motivators of use. Clinician's must assess and treat these problems, thus reducing the need for patients to self-medicate with cannabis, and therefore reducing the risk of relapse.
\end{abstract}

Key words: antipsychotic side-effects, self-medication, cannabis, psychosis.

\section{Australian and New Zealand Journal of Psychiatry 2006; 40:570-574}

Cannabis is increasingly recognized as a drug that may increase the risk of depression [1] and precipitate or exacerbate psychosis among predisposed individuals [24]. People with psychotic illnesses have a high rate of cannabis use $[5,6]$ and it is therefore important to understand why subjects with psychosis specifically use cannabis.

David Schofield, Research Assistant (Correspondence)

University of Sydney, Psychological Medicine, Royal North Shore Hospital, St Leonards, New South Wales 2065, Australia. E-mail: dschofie@nsccahs.health.nsw.gov.au

Chris Tennant, Head of Department; Louise Nash, Senior Lecturer; Alison Cornish, Research Assistant; Coletta Hobbs, Research Assistant; Gail Brennan, Research Assistant

University of Sydney, Psychological Medicine, Sydney, Australia

Louisa Degenhardt, Senior Lecturer

University of New South Wales, ENDARC, Sydney, Australia

Received 6 September 2005; revised 30 October 2005; accepted 9 November 2005 .
A number of studies have explored self-reported reasons for substance use in patients with psychotic disorders [6-8]. Regardless of the substance used, the results indicate three main motives for use [7]: (i) to relieve dysphoria, for example, 'to relax' [8]; (ii) for social reasons [7]; and (iii) for intoxication or to enhance positive mood [6]. A minority reported two additional possible motives related to relieving positive psychotic symptoms, for example, so as to 'decrease suspiciousness' [9] and decreasing the side-effects of medication [8].

The proposition that antipsychotic side-effects are a motivator for cannabis use has not been comprehensively examined. Dixon and colleagues (1991) asked subjects if they had used cannabis 'to decrease side-effects of medication' [8]. However, interpretation of this question is limited because it makes the assumption that subjects know which symptoms are associated with medication side-effects. In this regard there has been no detailed examination of the subjective experience of these 
side-effects, the degree of discomfort experienced and whether this discomfort is consciously self-medicated with cannabis.

\section{Current study}

This paper presents the results of a study of adults with schizophrenia spectrum disorders who have used cannabis in the past 6 months. The study aimed to examine (i) subjective discomfort from a comprehensive list of antipsychotic side-effects; (ii) medication side-effects reported to motivate cannabis use; (iii) psychological and social reasons for cannabis use; (iv) the relationship between the amount and frequency of cannabis used and (a) reported symptom discomfort and (b) the reasons given for use; and (v) reported efficacy of cannabis use in achieving desired effects.

\section{Method}

\section{Sample}

English-speaking men and women aged 16-50 years, with a diagnosis of schizophrenia, schizophreniform disorder or schizoaffective disorder were eligible for inclusion in the study. Only those who reported using cannabis in the 6 months prior to recruitment were included. All patients were currently being treated in community mental health centres in Northern Sydney.

\section{Study variables}

\section{Demographic variables}

Variables assessed included age, gender and highest educational achievement.

\section{Illicit drug use history}

Illicit drug use variables included the ages of first and regular cannabis use, monthly frequency of use, monthly quantity used, modes of all drugs used and other drug information (Table 1).

\section{Clinical variables}

Psychiatric diagnosis. All participants in this study had been diagnosed with a schizophrenia spectrum disorder by a hospital or community psychiatrist. Patients with bipolar disorder and those who admitted use of intravenous amphetamines in the previous 4 months were excluded.

\begin{tabular}{|lr|}
\hline \multicolumn{2}{|c|}{ Table 1. Drug use history $(n=101)$} \\
\hline Reported drug use \\
Last cannabis use \\
Past day & $\%$ \\
1-6 days ago & 24 \\
1-4 weeks ago & 19 \\
No cannabis used in the last month & 26 \\
Frequency of cannabis use in the past month & 31 \\
More than once daily & 16 \\
Daily & 5 \\
Between once/week and once/day & 17 \\
Less than weekly & 31 \\
No use & 31 \\
Amount of cannabis used per week & \\
More than $7 \mathrm{~g}$ & 7 \\
3-7 g & 11 \\
1-3 g & 14 \\
Less than $1 \mathrm{~g}$ & 37 \\
No current use & 31 \\
Method of use & \\
Joint & \\
'Bong' & 13 \\
Both & 45 \\
& 12 \\
\hline
\end{tabular}

Psychotropic medication. Subjects were asked the name dose of prescribed psychotropic medication, adherence to treatment and their daily self-medication regime. Anti-psychotic medication was characterized as typical or atypical.

\section{Reasons for cannabis use questionnaire}

This 25-item self-completion instrument was developed by Dickson (1991) to assess the reasons for drug use in patients with schizophrenia. The dimensions include dysphoria relief, improving sleep, relief of positive psychotic symptoms, enhancement of positive feelings, reduction of medication side-effects and social motives.

\section{Psychosis and Drug Abuse Scale (PADAS)}

The PADAS was developed for this study to assess possible subjective medication side-effects and whether cannabis was used to selfmedicate these symptoms. This instrument has 30 items derived from the UKU Side-effects Scale [10]. The UKU was developed to provide a comprehensive measure of adverse antipsychotic side-effects, categorized as psychic, neurologic, autonomic or other undifferentiated [10].

The items of the UKU were modified to produce a self-rated version, using the scale developed by Buise (1992) [11]. Side-effects listed in the PADAS were rated to assess the degree of discomfort experienced on a four-point Likert scale ranging from $0=$ absent, 1 = slight, 2 = considerable to $3=$ extreme. Participants also indicate whether they have used either cannabis or alcohol to try to relieve each symptom of discomfort using a yes/no/unsure scale. Reliability 
estimates by Cronbach alpha $(r=0.92)$ indicates a very good level of internal consistency.

It is important to note that for some of these symptoms (e.g. trembling), there is no way of determining if the origins are medication side-effects, side-effects of illicit drug use or part of the mental disorder process.

\section{Cannabis use effects survey}

The Cannabis Use Effects Survey was adapted from the 'Assessment of cannabis use' [12] to assess positive and negative effects of cannabis use. Participants report the frequency of each effect (e.g. helps me sleep) on an always/often/usually/sometimes/never Likert scale.

\section{Analysis strategy}

Descriptive analysis was conducted of self-reported symptoms, level of symptom distress and reasons for cannabis use. The rates of cannabis use (amount and frequency) were compared.

\section{Results}

\section{Participant characteristics}

One hundred and one eligible patients completed the baseline interview and questionnaires. The mean age was 25 years and $61 \%$ were on sickness benefits (median duration of 6 months). The vast majority was single men (89\%); most lived with others in independent accommodation and; $68 \%$ completed high school (year 12 ) and only $23 \%$ had paid work.

\section{Illicit drug use}

Participant's drug use history is shown in Table 1. Most started using cannabis in mid adolescence (median age 15 years), with regular use at 17 years and $69 \%$ reporting cannabis use in the past month. A considerable number reported using tobacco $(88 \%)$, alcohol (85\%) and caffeine $(87 \%)$ in the past month. Eleven per cent had used nonintravenous amphetamines, $9 \%$ used ecstasy, $3 \%$ cocaine and $2 \%$ nonintravenous opioids.

Mean age of onset of first psychotic symptoms was reported to be 19 years $(\mathrm{SD}=5$ years) and age of first mental health contact was 20 years $(\mathrm{SD}=5$ years). Eighty-one per cent had been hospitalized with a median of two admissions (range: 0-14). Fifty-five per cent of the most recent hospitalizations were involuntary.

\section{Discomfort from antipsychotic side-effects}

The mean level of discomfort experienced for each antipsychotic side-effect is shown in Table 2. Generally the most commonly occurring side-effects were also rated to be the most distressing. Analysis of the items clustered in the UKU subfactors showed that the psychic sideeffects were most distressing and more frequently associated with motivating cannabis use.

\section{Side-effects reported to motivate cannabis use}

With the exception of poor memory and sleepiness/sedation, the more distressing and common side-effects had the greatest link with cannabis use. Inner unrest/agitation (47\%) and difficulty sleeping (43\%) were the two symptoms most commonly 'self-medicated' with cannabis. Participants reported cannabis use to relieve muscle tension in $31 \%$, to assist lack of emotions or feeling for others in $25 \%$, for sleepiness/sedation in $25 \%$, for dreaming/nightmares in $22 \%$, for tiredness/lack of energy in $22 \%$ and for trembling/shaking in $22 \%$.

Analysis of other general reasons given for use showed cannabis was most commonly used to relax (86\%), as something to do with friends $(81 \%)$ and for relieving boredom (79\%). Approximately half of the sample used cannabis to improve sleep (58\%) and to reduce anxiety (49\%), while 39\% reported using cannabis to feel good about oneself. Only a small proportion reported using cannabis to either reduce medication side-effects (15\%) or to reduce positive symptoms of psychosis, specifically to decrease voices (11\%) and reduce paranoia (8\%).

\section{Patient reported efficacy of cannabis use}

Overall, subjects affirmed the efficacy of their cannabis use in relation to the most common reasons for use. On average, subjects reported that cannabis usually reduced their anxiety, and made them feel good, sleep better, experience less feelings of depression and relieve boredom between usually and often. However, in contrast subjects also reported that sometimes cannabis actually made them feel anxious and depressed. Subjects reported that sometimes cannabis 'helps me socialise'.

\section{The relationship between cannabis use and medication side-effects}

A significant moderate correlation was found between the amount and frequency of cannabis used, and average discomfort from PADAS symptoms ( $r=0.39$ and 0.49 respectively). Considering medication side-effects that were reported to be most common and causing greatest distress, the level of distress due to lack of emotions or feelings for others was moderately correlated with amount $(r=0.32, \mathrm{p}=0.03)$ and frequency of cannabis use $(r=0.29, \mathrm{p}=0.05)$. Muscle tension was moderately correlated with the amount of THC used $(r=0.30$, $\mathrm{p}=0.04)$, and difficulty sleeping $(r=0.32, \mathrm{p}=0.03)$ and trembling/ shaking $(r=0.33, \mathrm{p}=0.01)$ were moderately correlated with frequency of use.

Further analysis of these four side-effects was conducted comparing those experiencing distress with those reporting no distress. There was a trend of greater cannabis use (amount and frequency) when patients experienced distress due to these side-effects. These analyses were less sensitive than the correlation analysis, and the difference in cannabis use coming closest to significance was for lack of emotions or feelings 
Table 2. PADAS side-effects, reported discomfort and associated cannabis use $(n=44)$

\begin{tabular}{|c|c|c|c|}
\hline $\begin{array}{l}\text { PADAS } \\
\text { side-effect }\end{array}$ & $\begin{array}{c}\text { Present } \\
\%\end{array}$ & $\begin{array}{c}\text { Mean } \\
\text { level of } \\
\text { discomfort (SD) }\end{array}$ & $\begin{array}{l}\text { Per cent symptomatic } \\
\text { patients using cannabis } \\
\text { for side-effects } \%\end{array}$ \\
\hline 1. Tiredness, lack of energy & 88 & $1.8(1.0)$ & 22 \\
\hline 2. Inner unrest/agitation & 80 & $1.6(1.0)$ & 47 \\
\hline 3. Sleepiness/sedation & 72 & $1.3(1.0)$ & 25 \\
\hline 4. Poor memory & 68 & $1.4(1.2)$ & 6 \\
\hline 5. Dreaming/nightmares & 66 & $1.3(1.0)$ & 22 \\
\hline 6. Difficulty sleeping & 66 & $1.4(1.2)$ & 43 \\
\hline 7. Weight gain & 61 & $1.1(1.1)$ & 10 \\
\hline 8. Increased/decreased salivation & 58 & $1.0(1.1)$ & 4 \\
\hline 9. Trembling/shaking & 56 & $0.9(1.0)$ & 22 \\
\hline 10. Muscle tension & 54 & $1.0(1.0)$ & 31 \\
\hline 11. Lack of emotions/feelings for others & 53 & $1.0(1.1)$ & 25 \\
\hline 12. Involuntary bodily movements & 51 & $0.8(0.9)$ & 18 \\
\hline 13. Dizziness & 50 & $0.8(0.9)$ & 6 \\
\hline 14. Bodily rigidity & 49 & $0.8(1.0)$ & 18 \\
\hline 15. Increased sweating & 48 & $0.9(1.1)$ & 12 \\
\hline 16. Nausea/vomiting & 46 & $0.7(1.0)$ & 10 \\
\hline 17. Constipation & 43 & $0.7(1.0)$ & 10 \\
\hline 18. Heart palpitations & 42 & $0.7(0.9)$ & 12 \\
\hline 19. Difficulty seeing close objects clearly & 42 & $0.8(1.0)$ & 6 \\
\hline 20. Sexual problems & 40 & $0.7(1.1)$ & 16 \\
\hline 21. Slowed or reduced movements & 37 & $0.7(1.0)$ & 8 \\
\hline 22. Diarrhoea & 36 & $0.6(0.9)$ & 6 \\
\hline 23. Itchiness & 33 & $0.5(0.8)$ & 0 \\
\hline 24. Skin sensitivity to sun & 33 & $0.6(1.0)$ & 2 \\
\hline 25. Skin sensations & 32 & $0.5(0.8)$ & 4 \\
\hline Headaches & 30 & $0.6(0.9)$ & 12 \\
\hline Difficulty urinating & 21 & $0.3(0.8)$ & 2 \\
\hline Breast changes & 15 & $0.2(0.7)$ & 0 \\
\hline 29. Change in menstruation & 10 & $0.2(0.6)$ & 4 \\
\hline 30. Epileptic seizures & 2 & $0.0(0.2)$ & 2 \\
\hline
\end{tabular}

for others (amount, $\mathrm{p}=0.04$; frequency, $\mathrm{p}=0.02$ ). Those experiencing lack of emotions or feelings for others, used more cannabis $(6.9 \mathrm{~g}$, compared with $1.8 \mathrm{~g}$ ) in the past month, and used more frequently ( 8.7 days month ${ }^{-1}$, compared with 1.8 days month $^{-1}$ for those not experiencing this symptom).

Cannabis use was similarly compared between those who reported the side-effect as a motivator for use, with those who did not. The consistent trend was for a greater amount and frequency of cannabis use when the side-effects were motivators. Although not quite significant $(\mathrm{p}=0.02$, Bonferoni adjusted alpha $=0.01)$ there was a trend towards greater frequency of cannabis use (11.9 days month $^{-1} v s$ 5.0 days) in subjects using cannabis to relieve trembling/shaking.

\section{Discussion}

Cannabis use is more common in people with psychosis than the general population [13]. Similarly, cannabis is recognized as a drug that may precipitate or exacerbate the symptoms of a psychotic illness [2]. Reducing can- nabis use in patients with psychosis is therefore of great clinical importance. In addition, an issue worthy of consideration is that these patients may be self-medicating not only symptoms of their illness but also medication side-effects.

Consistent with similar research [6-8], the most common reasons given for cannabis use were to reduce boredom $(86 \%)$, something to do with friends $(81 \%)$ and to improve sleep (58\%). The next most common reason was to reduce anxiety (49\%).

Anxiety may account for the majority of the most common and distressing symptoms reported to motivate cannabis use: Inner-unrest/agitation, difficulty sleeping, muscle tension, dreaming/nightmares and trembling shaking. These symptoms may be antipsychotic sideeffects, but could also be expected of a person experiencing anxiety, psychosis and/or depression.

The three other possible side-effects emerging as particularly associated with cannabis use, were lack of 
emotions/feelings for others, tiredness/lack of energy and sleepiness/sedation. Together with the most common motivator, 'boredom', these symptoms are common to the negative symptoms of psychosis or indeed depression. Consistent with this proposition is a trend towards greater cannabis use in subjects who reported that cannabis 'takes away the feelings of depression', and in those who reported that they were distressed by lack of feelings/emotions for others, tiredness/lack of energy and sleepiness/sedation.

In relation to specific problems associated with psychosis, only a tenth reported using it to reduce positive symptoms. The most significant symptom motivators of cannabis may have more to do with anxiety and depression which may be related to the disease process rather than to either positive psychotic symptoms or antipsychotic side-effects (unless antipsychotics cause anxiety/ depression symptoms).

This was the first study to comprehensively examine subjective experiences of medication side-effects and link these to reasons for cannabis use. It relied on selfreport data alone because an understanding of personal (subjective) reasons why patients with psychotic disorders use cannabis is crucial in reducing use and abuse in these patients. This is particularly the case when considering interventions that may assist with reducing cannabis use among this group.

The symptoms that motivate cannabis use include social isolation, lack of emotion or feeling for others, lack of energy, difficulty sleeping, depression, anxiety, agitation and tremor or shaking. These symptoms may occur as part of the psychotic illness, be due to anxiety/ depressive illnesses, and occasionally may be sideeffects of medication. Cannabis in this population can trigger relapse therefore it is important to reduce these motivators. A careful assessment of the presence of motivators, and appropriate medication strategies to target symptoms and reduce side-effects, is essential. Drug education and interventions to reduce the use of cannabis may also be useful. Recent evidence has suggested that brief motivational interventions may assist in the reduction of substance use among persons with psychosis [14].

Illness and side-effects of medication are not the only reasons for using cannabis. Many of the subjects reported using cannabis as an aid to socialization. Cannabis usage to an extent confers membership in the cannabis user network and may be an important way for people with psychosis to maintain social contacts. Cannabis may also enable greater individual social acceptability as the effects of cannabis can mask psychotic symptomatology. This suggests that interventions to assist in ceasing cannabis use should include social skills training and help to establish new social networks.

\section{Conclusion}

The central findings to emerge from this study is that positive symptoms of psychosis are not the primary reason for patients with schizophrenia spectrum disorders to use cannabis, nor indeed are types of medication sideeffects that have no association with anxiety, depression or negative psychotic symptoms. What does emerge is that reducing boredom, improving socialization, and a cluster of symptoms that may be related to either medication side-effects or the illness itself, are important motivators for cannabis use. Clinician's must assess and treat these symptoms, thus reducing the need for patients to self-medicate with cannabis, and therefore reducing the risk of relapse.

\section{References}

1. Patton GC, Coffey C, Carlin JB, Degenhardt L, Lynskey M, Hall W. Cannabis use and mental health in younger people: cohort study. British Medical Journal 2002; 325:1195-1198.

2. Arseneault L, Cannon M, Witton J, Murray R. Causal association between cannabis and psychosis: examination of the evidence. British Journal of Psychiatry 2004; 184:110-117.

3. McKay DR, Tennant CC. Is the grass greener? The link between cannabis and psychosis. Medical Journal of Australia 2000; 172:284-286.

4. Degenhardt L, Hall W, Lynskey M. Testing hypotheses about the relationship between cannabis use and psychosis. Drug and Alcohol Dependence 2003; 71:31-48.

5. Barbee JG, Clark PD, Crapanzo MS, Heintz GC, Kehoe CE Alcohol and substance abuse among schizophrenic patients presenting to an emergency psychiatry service. Journal of Nervous and Mental Disease 1989; 177:400-417.

6. Fowler I, Carr V, Carter N, Lewin T. Patterns of current and lifetime substance use in schizophrenia. Schizophrenia Bulletin 1998; 24:443-455.

7. Spencer C, Castle D, Michie PT. Motivations that maintain substance use among individuals with psychotic disorders. Schizophrenia Bulletin 2002; 28:233-247.

8. Dixon L, Haas G, Weiden PJ, Sweeney J, Frances AJ. Drug abuse in schizophrenic patients: clinical correlates and reasons for use. American Journal of Psychiatry 1991; 148:224-230.

9. Test MA, Wallisch LS, Allness DJ, Ripp K. Substance use in young adults with schizophrenic disorders. Schizophrenia Bulletin 1989; 15:465-476.

10. Lingjaerde O, Ahlfors UG, Bech $\mathrm{P}$ et al. The UKU side effect rating scale. Acta Psychiatrica Scandinavica 1987; 76: 85-94.

11. Buis W. Patients' opinions concerning side effects of depot antipsychotics. American Journal of Psychiatry 1992; 149:844845 .

12. Rolfe T, McGorry P, Cocks J, Yuen H, Longley T, Plowright D.Cannabis use in first episode psychosis, incidence and short term outcome. Inaugural Cannabis and Psychosis Conference Final Papers, Melbourne, 1999.

13. Degenhardt L, Hall W. The association between psychosis and problematical drug use among Australian adults: findings from the National Survey of Mental Health and Well-Being. Psychological Medicine 2001; 31:659-668.

14. Kavanagh DJ, Young R, White A et al. A brief motivational intervention for substance misuse in recent-onset psychosis. Drug and Alcohol Review 2004; 23:151-155. 